\title{
Frequency-dependent signal transfer at the interface between electrogenic cells and nanocavity electrodes
}

\author{
Manuel Schottdorf, ${ }^{*}$ Boris Hofmann, ${ }^{\dagger}$ Enno Kätelhön, Andreas Offenhäusser, and Bernhard Wolfrum ${ }^{\ddagger}$ \\ Institute of Bioelectronics (PGI-8/ICS-8), Forschungszentrum Jülich GmbH, D-52425 Jülich, Germany and \\ JARA - Fundamentals of Future Information Technologies, Jülich-Aachen Research Alliance, Germany
} (Received 2 December 2011; revised manuscript received 24 February 2012; published 29 March 2012)

\begin{abstract}
We present a model to describe the response of chip-based nanocavity sensors during extracellular recording of action potentials. These sensors feature microelectrodes which are embedded in liquid-filled cavities. They can be used for the highly localized detection of electrical signals on a chip. We calculate the sensor's impedance and simulate the propagation of action potentials. Subsequently we apply our findings to analyze cell-chip coupling properties. The results are compared to experimental data obtained from cardiomyocyte-like cells. We show that both the impedance and the modeled action potentials fit the experimental data well. Furthermore, we find evidence for a large seal resistance of cardiomyocytes on nanocavity sensors compared to conventional planar recording systems.
\end{abstract}

DOI: 10.1103/PhysRevE.85.031917

PACS number(s): 87.80.Jg, 87.85.dh, 87.85.Qr

\section{INTRODUCTION}

The electrophysiological activity of individual cells is generally observed using the patch clamp technique [1]. This technique and related methods [2] yield very high signal-to-noise ratios and are ideally suited to study the activity of ion channels. However, these techniques are limited with regard to long-term measurements and the number of recording units in a single experiment. Extracellular electrophysiological methods, on the other hand, yield weaker signals but allow long-term and multisite recordings of cellular networks due to reduced interference with cell viability. A well-established method for extracellular measurements relies on microelectrode arrays (MEAs) [3-6]. Fields of application for in vitro MEA systems include pharmacological highthroughput screening, cell-based biosensors, and research on information processing in neuronal networks [7-13]. During the last years, the integration of complementary metal oxide semiconductor technology (CMOS) with MEAs has led to the development of very high-density recording units, opening up new opportunities for the investigation of communication in cellular networks $[14,15]$. Nevertheless, the simple fabrication of low-impedance and high-spatial-resolution electrodes still remains an experimental challenge. Attempts to lower the impedance, including the use of advanced interface materials [16-18] and patterning of metal electrodes [19-22], have led to promising results. However, they often require sophisticated techniques of production or lack mechanical stability and longevity. We have recently introduced a sensor design which includes a fluid-filled nanocavity covering a metal electrode $[23,24]$. This approach decreases the impedance of the sensor due to an increase of the electrode-electrolyte interface area. The spatial resolution of such nanocavity sensors is preserved

\footnotetext{
*Present address: Institut für Theoretische Physik, Universität Würzburg, Am Hubland, D-97074 Würzburg, Germany; mschottdorf@physik.uni-wuerzburg.de

${ }^{\dagger}$ Present address: Aesculap AG, Am Aesculap Platz, D-78532 Tuttlingen, Germany.

†.wolfrum@fz-juelich.de
}

by the small aperture which connects the nanofluidic cavity with the chip's surface. Modeling the signal transduction at the cell-sensor interface is important to optimize the system's design and to understand how a cell's action potential is related to the measured signal [25-27]. In this paper we present a model to describe the impedance and the cell-sensor coupling of a nanocavity electrode. Cell-sensor interfaces have been studied in the equivalent circuit approach, namely the area and the point-contact model and by solving the Poisson-Boltzmann-Planck equation [28-31]. To investigate the nanocavity sensor's response we will rely on the equivalent circuit approach. We calculate the response of the sensors to a simulated action potential and compare the result with signals of HL-1 cells measured with nanocavity electrodes. The HL-1 cell line has been derived by Claycomb et al. from mouse cardiomyocyte tumor cells (AT-1) [32]. It exhibits spontaneous contraction and firing of action potentials in cell culture. Thus it is a well suited model system to study bioelectronic devices [33,34]. We demonstrate the validity of our model and we show evidence for an improved seal resistance of HL-1 cells on nanocavity sensors compared to planar cell-chip interfaces.

\section{EXPERIMENTAL METHODS}

We fabricated nanocavity electrode arrays by standard optical lithography and sacrificial etching of chromium layers. A detailed description of the fabrication process is given elsewhere [24]. Briefly, gold electrodes, feed lines, bond pads, and chromium sacrificial layers were fabricated on siliconsilicon oxide wafers using a two-step optical lithography process. The whole structure was insulated against electrolytes via the plasma-enhanced chemical vapor deposition of $800 \mathrm{~nm}$ silicon oxide and silicon nitride layers. The apertures and bond pads of the device were opened by reactive ion etching. We used sacrificial layer etching of the chromium layer to open the nanocavity covering the gold electrode. The nanocavities above the electrodes have a diameter of $30 \mu \mathrm{m}$ and a height of $70 \mathrm{~nm}$. The size of the central aperture is $5 \mu \mathrm{m}$ in diameter and $800 \mathrm{~nm}$ in height. HL-1 cells, kindly 
provided by William Claycomb, represent a hybrid between embryonic and adult myocytes. They form confluent layers and are connected via gap junctions. HL-1 cells generate action potentials spontaneously, pervading the entire cell population [32]. We have cultured cells and seeded them onto chips as previously described [24]. Briefly, the cells were kept in a T25 flask with a supplemented Claycomb medium (supplemented with $10 \% \mathrm{FBS}, 100 \mu \mathrm{gml}^{-1}$ penicillin-streptomycin, $0.1 \mathrm{mM}$ norepinephrine, and $2 \mathrm{mM}$ L-glutamine) in an incubator at $37^{\circ} \mathrm{C}$. After coalescing of the the cells to a confluent layer they were passaged using standard procedures. Approximately 3000 cells were plated in $50 \mu$ l of the medium onto the nanocavity chip. After $4 \mathrm{~h}$ of incubation, the chips were topped up with fresh medium. The medium was changed daily and the chips were measured after three to four days when cells had reached confluence. Cellular activity was recorded from the potential between the nanocavity electrodes versus an extracellular $\mathrm{Ag}-\mathrm{AgCl}$ reference electrode using a home-built 64-channel voltage amplifier system. The amplifier consists of a preamplifier and a main stage with a total amplification factor of 1022. Electrode signals were sampled with a rate of $10 \mathrm{kHz}$ with the MED64 CONDUCTOR 3.1 software. Impedance measurements were performed with a potentiostat Autolab, PGSTAT 300.

\section{SENSOR IMPEDANCE}

The cell-sensor interface for dish electrodes has been studied in one dimension [35] as well as in two dimensions $[28,30]$. Common models to calculate the electrical properties of a given cell-sensor interface are the point-contact and the area-contact models [26,30,31,35,36]. Both models represent elements of the membrane, the electrode, and the chip either as resistors or capacitors. The area-contact model dissects the cell and the chip into infinitesimal units, which are related to each other by Kirchhoff's laws taking the spatial distribution of electrical elements into account. The point-contact model is an approximation of the area-contact model. It ignores the spatial dependency of voltages and currents. To describe the nanocavity device we will use a combination of both the area- and the point-contact models. We describe the cavity by a continuous two-dimensional system and the rest of the cell-sensor interface by a point-contact model. The description of the cavity which we introduce is similar to Heaviside's Bessel cable in two dimensions. This model has been used to describe the signal propagation along cellular membranes [37] and between cells in a thin layer [38,39]. An electrode immersed into an aqueous solution can be seen as an interface, which separates electron charge carriers from ionic charge carriers. Given that applied voltages are small enough to avoid electrochemical processes at the electrode, the interface can be described by a constant phase element

$$
Z(\omega)=\frac{Z_{0}}{(i \omega)^{\beta}} .
$$

$Z_{0}$ is a constant that depends on the electrode material, the exposed surface area, and the environment. For systems with gold-platinum electrodes and water the value of $\beta$ is around 0.8 or $0.9[40,41]$. The alleged reason for this power-law behavior is a nonsmooth and fractal surface topology of the electrode material [42-44]. With a model of the cell-sensor interface (Fig. 1), we can write an equation for an infinitesimal element in the cavity [Fig. 1(c)]

$$
Z_{\text {cav }}+d Z_{\text {cav }}=\left(\frac{1}{Z_{\text {cav }}}+\frac{1}{d X}\right)^{-1}+d R,
$$

where $d X$ and $d R$ are described at position $s$ in a cylindrical system. $s$ is the distance to the entry point of the cavity, which itself is located at a radius $r_{0} . Z_{\text {cav }}(s)$ is the impedance of a ring electrode covered with a nanocavity with inner radius $r_{0}$ and outer radius $r_{0}+s$. We can also write

$$
d R=\rho \frac{d s}{2 \pi\left(s+r_{0}\right) h} \equiv \frac{a}{s+r_{0}} d s,
$$

where $h$ is the height of the nanocavity, $\rho$ is the resistivity of the electrolyte, and $a$ is a constant proportional to the inverse height. Also

$$
d X=\frac{1}{2 \pi C_{h}\left(s+r_{0}\right)(i \omega)^{\beta} d s} \equiv \frac{b(\omega)}{\left(s+r_{0}\right) d s},
$$

where $C_{h}$ is the quasicapacitance per unit area and $\beta$ the exponent of the constant phase element. We introduce a new variable $b(\omega)$ to include these parameters and to simplify the equation. Equation (2) can be expressed along an infinitesimal element ds as a differential equation

$$
Z_{\text {cav }}^{\prime}(s)=\frac{a}{s+r_{0}}-\frac{Z_{\text {cav }}(s)^{2}\left(s+r_{0}\right)}{b},
$$

which describes the impedance of a nanocavity electrode with an inner radius $r_{0}$ as a function of $s$. The boundary condition is $Z_{\text {cav }}(s=0) \rightarrow \infty$ : an infinitesimally thin electrode ring has an infinite impedance. The solution to the differential equation is

$$
Z_{\mathrm{cav}}(s)=\frac{\sqrt{a b}}{\left(s+r_{0}\right)} \frac{-i I_{1}\left(\sqrt{\frac{a}{b}} r_{0}\right) Y_{0}\left[i \sqrt{\frac{a}{b}}\left(s+r_{0}\right)\right]+Y_{1}\left(i \sqrt{\frac{a}{b}} r_{0}\right) I_{0}\left[\sqrt{\frac{a}{b}}\left(s+r_{0}\right)\right]}{I_{1}\left[\sqrt{\frac{a}{b}}\left(s+r_{0}\right)\right] Y_{1}\left(i \sqrt{\frac{a}{b}} r_{0}\right)-I_{1}\left(\sqrt{\frac{a}{b}} r_{0}\right) Y_{1}\left[i \sqrt{\frac{a}{b}}\left(s+r_{0}\right)\right]},
$$

where $I_{n}$ are modified Bessel functions of the first kind of order $n, Y_{n}$ are Neumann functions (Bessel functions of the second kind) of order $n$ (see Appendix A for the derivation and Appendix B for a discussion of the solution's asymptotic 
(a)

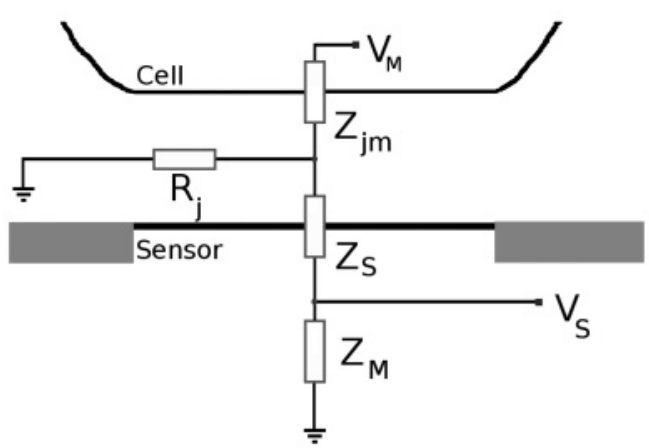

(b)

(c)

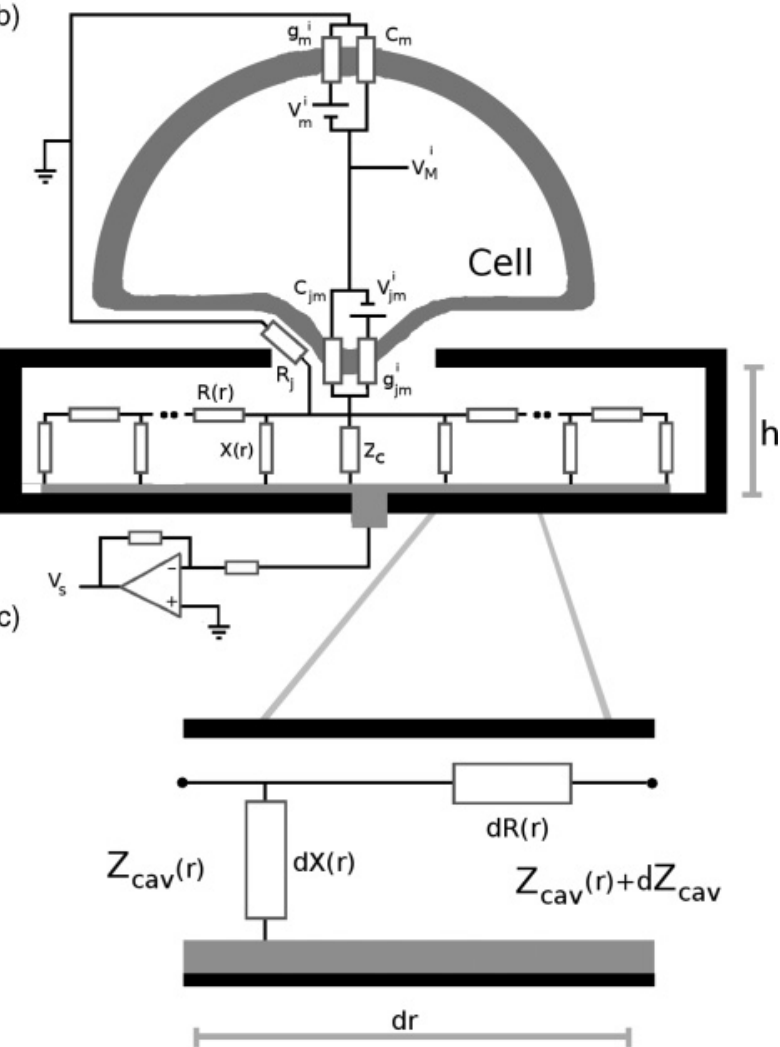

FIG. 1. The cell-sensor interface. (a) The coupling of the cell to the measurement system is shown. $V_{M}$ is the cell's potential in reference to extracellular space. $Z_{j m}$ describes the impedance of the cell membrane covering the aperture of the sensor, $R_{j}$ is the seal resistance, scaling the leakage current from the junction to the electrolyte. $Z_{S}$ is the impedance of the nanocavity sensor and $Z_{M}$ is the impedance of the measurement setup. $V_{S}$ is the voltage measured with the voltage amplifier. (b) The system is shown in more detail. The cell with Hodgkin-Huxley elements embedded in the membrane sits on the nanocavity electrode. $V_{m}^{i}$ is the Nernst voltage of the free membrane of the ion type $i, g_{m}^{i}$ is the membrane conductivity per unit area for this specific ion. $c_{m}$ is the capacitance per unit area of the cell membrane. Analogously, $V_{j m}^{i}$ is the Nernst voltage of the attached membrane, $g_{j m}^{i}$ the attached membrane's conductivity, and $c_{j m}$ the capacitance. Also shown is a voltage amplifier attached to the electrode. $Z_{c}$ is the impedance of the central spot of the microelectrode and $R_{j}$ is the leak resistance within the junction. $h$ is the height of the nanocavity. (c) A segment of the nanocavity in the radial direction is shown. $d R$ is the ohmic resistivity of the electrolyte and $d X$ the constant phase impedance of the electrode. Both $R(r)$ and $X(r)$ are functions of the distance $r$ to the center of the electrode. behavior). To calculate the total impedance of the sensor $Z_{S}$ we have to add the impedance of the central spot

$$
Z_{S}=\left(\frac{1}{Z_{\mathrm{cav}}\left(r_{\max }-r_{0}\right)}+(i \omega)^{\beta} C_{h} \pi r_{0}^{2}\right)^{-1} .
$$

$r_{0}$ is the radius of the central opening, $r_{\text {max }}$ the total electrode radius. This equation describes the impedance of a nanocavity microelectrode exclusively as a function of the geometry, the conductivity of the electrolyte, and the parameters of the electrode's impedance. We can use it to fit the experimental data (Figs. 2 and 3).

\section{SIGNAL PROPAGATION}

\section{A. Propagation of action potentials}

Cells can generate action potentials due to voltagedependent ion channels embedded in their membranes. The total current density through the cell's membrane is the sum of capacitive and ionic current densities [30,45] and depends on the cellular potential $V_{M}$

$$
j_{m}=c_{m} \frac{d V_{M}}{d t}+\sum_{i} g_{m}^{i}\left(V_{M}-V_{0}^{i}\right),
$$

where $j_{m}$ is the current density through the membrane, $c_{m}$ the membrane capacitance per unit area, and $g_{m}^{i}$ the conductance with respect to the ion type $i$ per unit area. The equilibrium (Nernst) voltage of ion type $i$ is $V_{0}^{i}$. In the typical case without current clamp control, the ionic current through the membrane cancels the capacitive current. Thus, it is difficult to measure an extracellular signal in the vicinity of a membrane in Hodgkin-Huxley equilibrium. Inhomogeneities of the membrane attached to a sensor are a prerequisite for the recording of cellular activity [29]. Inhomogeneities of cardiac cells on sensors are discussed elsewhere in detail [33,34]. A scaled conductance of the ion channels in the attached



FIG. 2. Shown is a Bode plot of experimental data (symbols) and model fits (solid lines). The absolute value and the phase of the impedance as a function of the frequency are shown. The squares are measured values of the impedance. The circles are measured values of the phase. The solid lines are the absolute value and phase of Eq. (7). The microelectrode used for this measurement has a central opening with radius $r_{0}=2.5 \mu \mathrm{m}$ and a total radius of $r=15 \mu \mathrm{m}$. The theory curves are calculated for $a=6 \times 10^{5} \Omega, C_{h}=59 \mu \mathrm{Fcm}^{-2}$, and $\beta=0.89$. 


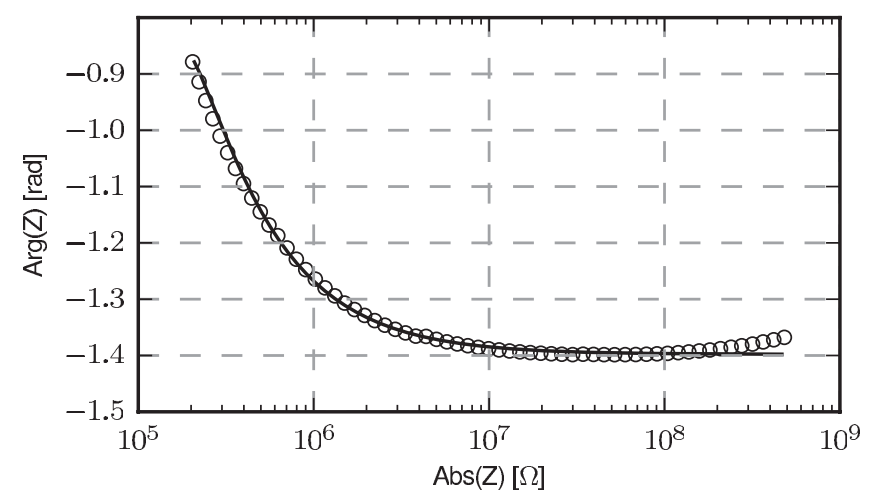

FIG. 3. Shown is a plot of the phase as a function of the absolute value of the impedance. We compare experimental data (circles) with a fit from Eq. (7) (solid line). The parameters are the same as in Fig. 2.

membrane by a factor $\chi_{i}$ where $i=\mathrm{Na}, \mathrm{K}, \mathrm{Ca}$

$$
g_{j m}^{i}=\chi_{i} g_{m}^{i}
$$

leads to

$$
j_{j m}=c_{m} \frac{d V_{M}}{d t}+\sum_{i} \chi_{i} g_{m}^{i}\left(V_{M}-V_{0}^{i}\right),
$$

which we will take as an ansatz to simulate the measured signals. Here we assume that the equilibrium Nernst potential within the cleft is identical to the free membrane potential $V_{0}^{i}$ and that the capacitance per unit area of the free membrane $c_{m}$ is identical to the capacitance per area of the attached membrane $c_{j m}$. Using the equivalent circuit in Fig. 1(a), the theoretical impedance function of the nanocavity sensor, Eq. (7), and an impedance of the voltage amplifier $Z_{M}$ consisting of a capacitance of $150 \mathrm{pF}$ and a resistance of $100 \mathrm{M} \Omega$ in parallel, we can calculate signals for any cell type given that the ion channel kinetics are known. We choose a model of human myocardial cells as an approximation for HL-1 cells [46]. It is a Hodgkin-Huxley model for 12 different ion channels consisting of 28 differential equations. We consider all of the sodium currents to scale with a common

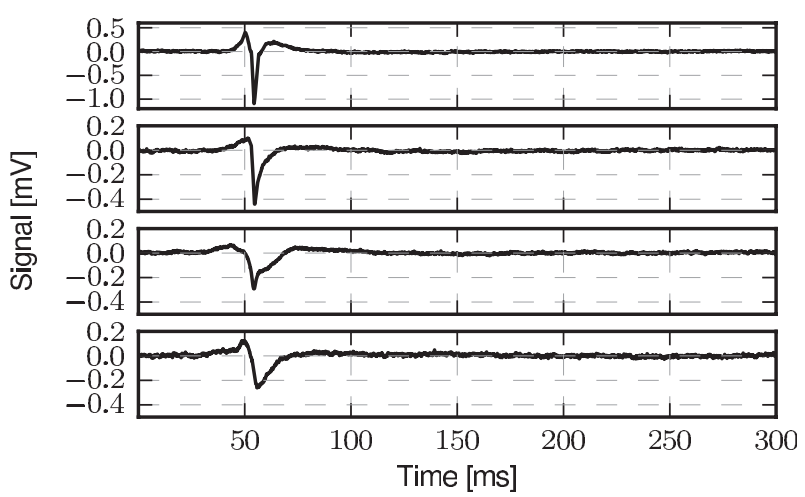

FIG. 4. The electrical activity of HL-1 cells growing on the surface of a chip with nanocavity microelectrodes has been recorded. We show four different signals, which are not only qualitatively but quantitatively different. The used microelectrodes are geometrically identical. They have a central opening with radius $r_{0}=2.5 \mu \mathrm{m}$ and a total radius of $r=15 \mu \mathrm{m}$. The measurements have been performed in Claycomb's medium against a $\mathrm{Ag}-\mathrm{AgCl}$ reference electrode.
$\chi_{\mathrm{Na}}$. The calcium currents with $\chi_{\mathrm{Ca}}$ and the potassium currents with $\chi_{\mathrm{K}}$. We have chosen a Gaussian stimulation signal $I_{\text {stim }}(t)$ to excite the action potential of the model cell. The model works with absolute currents. Estimating the radius of an HL-1 cell to be $7 \mu \mathrm{m}$ [47] and knowing the electrode opening radius of $2.5 \mu \mathrm{m}$ we assume $\chi_{\text {total }} \approx 5 \%$ of the total current $I_{\text {total }}$ through the membrane to be important for the signal

$$
I_{j m}=\chi_{\text {total }} I_{\text {total }} \text {. }
$$

To calculate signals we have to convolve the model action potential with the response function parametrized in time. However, it is simpler to multiply the Fourier-transformed action potential of the cell with the response function of the measurement system and to transform the result back. The free parameters for our model are the three scaling parameters $\chi_{i}$ and the seal resistance $R_{j}$. The amplifier and the sensor impedance are kept constant for all simulated signals. For comparison we measured a total number of 57 signals on five different chips, each with 36 nanocavity electrodes. We present an overview of the signals, which we measured (Fig. 4) and explain them within the framework of our model. The results of our simulation are shown in Fig. 5. Signal 1 has a large amplitude. We can find signals of similar magnitude and shape in about $10 \%$ of our data. They require a very strong sodium peak $\chi_{\mathrm{Na}}=2.7, \chi_{\mathrm{K}}=1.2$, and $\chi_{\mathrm{Ca}}=1.0$ and a large seal resistance $R_{j}=50 \mathrm{M} \Omega$. Similar signals are observed in field-effect transistor experiments with rat cardiac muscle cells as well $[33,34]$. The simulation of signal 2 requires a seal resistance of $R_{j}=30 \mathrm{M} \Omega, \chi_{\mathrm{Na}}=2.1, \chi_{\mathrm{K}}=\chi_{\mathrm{Ca}}=1.0$. The amplitude of this signal is smaller, especially the overshooting to positive voltages after the strong negative sodium peak. It is a typical signal which appears in about $50 \%$ of all cases. The typical amplitude is about $0.5 \mathrm{mV}$. Signal 3 can be modeled with $R_{j}=20 \mathrm{M} \Omega, \chi_{\mathrm{Na}}=1.35$, and $\chi_{\mathrm{K}}=1$. Here we have to split the calcium currents into two parts. The $L$-type calcium

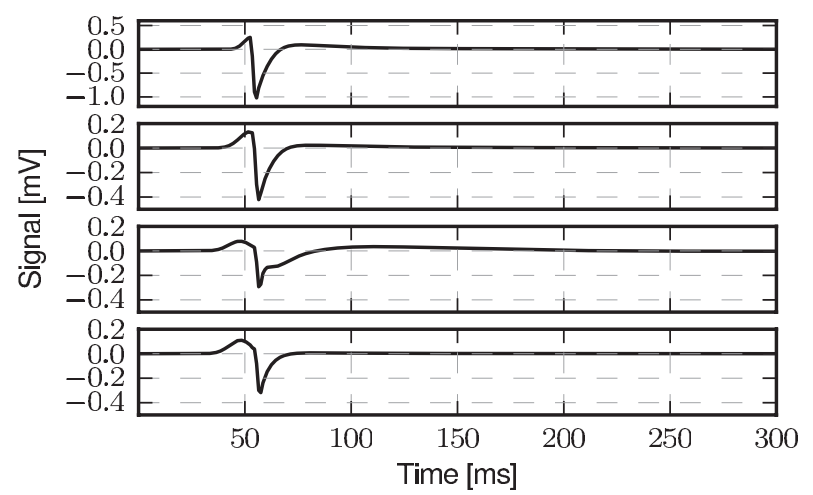

FIG. 5. Simulated signals for the same type of electrode as in Figs. 2 and 4. The presented signals have been calculated from the circuitry in Fig. 1 and a model for human myocardial cells [46]. Free parameters to reproduce the experimental data are the scaling of the ion channels $\chi_{i}$ and the quality of the seal between the cell and sensor measured with $R_{j}$. Signal 1 has been calculated with $R_{j}=50 \mathrm{M} \Omega$, $\chi_{\mathrm{Na}}=2.7$ and $\chi_{\mathrm{K}}=1.2 \chi_{\mathrm{Ca}}=1.0$. Signal 2 has been calculated with $R_{j}=30 \mathrm{M} \Omega, \chi_{\mathrm{Na}}=2.1, \chi_{\mathrm{K}}=1.0$, and $\chi_{\mathrm{Ca}}=1.0$. Signal 3 shows a leaky cell with $R_{j}=20 \mathrm{M} \Omega, \chi_{\mathrm{Na}}=1.35, \chi_{\mathrm{K}}=1$, and $\chi_{\mathrm{Ca}}^{2}=2.3$. The background calcium current is very strong $\chi_{\mathrm{Ca}}^{1}=14$. Signal 4 is calculated with $R_{j}=35 \mathrm{M} \Omega, \chi_{\mathrm{Na}}=1.3$, and $\chi_{\mathrm{K}}=\chi_{\mathrm{Ca}}=1$. 
current and sarcolemmal calcium pump current scale with $\chi_{\mathrm{Ca}}^{2}=2.3$, the background calcium current is quite strong $\chi_{\mathrm{Ca}}^{1}=14$. Very large inhomogeneities in the calcium-current contributions have been described previously [33,34]. Signals of this shape appear in about $10 \%$ of the measurements. This might be an indication of cell damage from the large leakage current. Signal 4 is a typical example of a weak signal with an amplitude around $0.3 \mathrm{mV}$ that appeared in $30 \%$ of our measurements. $R_{j}=35 \mathrm{M} \Omega, \chi_{\mathrm{Na}}=1.3, \chi_{\mathrm{K}}=\chi_{\mathrm{Ca}}=1$. Signals of this type have no visible overshoot after the sodium peak. The seal resistance for the simulated signals above are in the range of a few tens of $M \Omega$ s. Previously reported seal resistances for plain chips are a factor of 10 smaller $[33,48]$. So far, high seal resistances without active suction or penetration of the probe have only been shown for spine-shaped gold protrusions [49]. We explain the excellent sealing of the electrode by the small opening which can be covered completely by the cell. Electron microscopy studies of the cell-chip interface reveal that the cell membrane seals the opening very well [24]. It did not escape out attention that we have not found any signals which stand out with their potassium contributions although we find examples for strong calcium and strong sodium currents. The absence of potassium signals has been described by [33] as well.

\section{B. Influence of the geometry on signal propagation}

From the equation describing the nanocavity impedance, Eq. (7), we can study how changing the radius influences the impedance of the electrode and the signal transfer from the cell to the sensor. The signal transfer function of an attached cell can be calculated if the junction membrane impedance $Z_{j m}(\omega)$ is known. We use a linear model where this impedance consists of a parallel capacitance and a resistance which is described in detail in [30]. We want to ascertain the signal transfer function $h(\omega)$ which is in frequency space defined by

$$
V_{S}(\omega)=h(\omega) V_{M}(\omega)
$$

for different electrode sizes. Figure 6 shows the signal transfer function for sensors with electrode radii of 10,15 , and $50 \mu \mathrm{m}$.

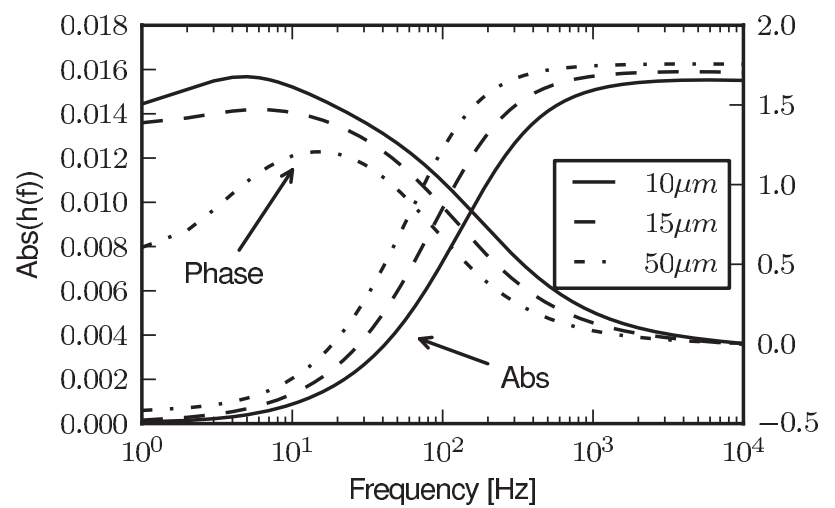

FIG. 6. Three response functions calculated for three different electrode radii. The graphs show the absolute values $|h(f)|$ and the phases $\arg (h(f))$ as indicated by the arrows. All other parameters, $C_{h}=59 \mu \mathrm{Fcm}^{-2}, a=6 \times 10^{5} \Omega, R_{\text {load }}=10^{8} \Omega, C_{\text {load }}=150 \mathrm{pF}$, $R_{j}=15 \mathrm{M} \Omega, \beta=0.89, R_{j m}=20 \mathrm{G} \Omega$, and $C_{j m}=2.5 \mathrm{pF}$ are kept constant.
The diameter of the inner opening is $5 \mu \mathrm{m}$. We can see that the nanocavity sensor system is a high pass filter with a step-like shape. The slope of the step depends weakly on the electrode diameter. The value to which $|h(\omega)|$ converges depends on the seal resistance, the sensor impedance, and the impedance of the measurement setup. Greater electrode diameters increase the maximum value of $|h(\omega)|$ and shift the cutoff frequency of the high pass filter toward smaller frequencies. In this sense, a larger electrode diameter improves the sensor performance at constant aperture size.

\section{CONCLUSION AND OUTLINE}

In summary, we have calculated the complex impedance of a nanocavity sensor, which is in good agreement with experimental data obtained from impedance spectroscopy measurements. Furthermore, we have presented a model for analyzing the signal transfer of cells growing on top of the sensor array. Action potentials have been calculated and compared with the measurements from HL-1 cells in vitro. We have confirmed the validity of our model and demonstrated the influence of the geometry of such devices. As a result of our analysis we find that the seal resistance between the cell and the chip is a factor of 10 greater than in the plain standard electrode system. Thus, cell signals can reliably be measured with a high spacial resolution. A next step is the application of our model to dual-electrode nanocavity devices [23]. These sensors feature a top electrode at the ceiling of the cavity and allow signal-correlation analysis, which will possibly result in an improved signal-to-noise-ratio for extracellular recording of action potentials.

\section{ACKNOWLEDGMENTS}

The authors thank William Claycomb for providing the cells, Rita Fricke for support with the cell culture, and Michael Pabst and Philipp Moritz for comments on the paper and support with the calculations. Manuel Schottdorf thanks Elitenetzwerk Bayern for financial support. The project is funded by the Helmholtz Young Investigator Program.

\section{APPENDIX A: DERIVATION OF THE IMPEDANCE EQUATION (6)}

Equation (5) is a Riccati equation of the form

$$
z^{\prime}(s)=f(s) z^{2}(s)+h(s),
$$

with $f(s)=-\left(s+r_{0}\right) / b$ and $h(s)=a /\left(s+r_{0}\right)$. The Riccati differential equation can be transformed into a second-order differential equation

$$
y^{\prime \prime}(s)-\frac{f^{\prime}(s)}{f(s)} y^{\prime}(s)+f(s) h(s) y(s)=0,
$$

such that $z(s)=-y^{\prime}(s) /[f(s) y(s)]$ is the solution of the original Riccati equation [50]. After this transformation we 




FIG. 7. This figure shows the error measure Eq. (B7) for the approximations Eq. (B4) (dashed line) and Eq. (B5) (solid line). Since $\lambda$ depends on $\omega$, the quality of the approximation Eq. (B4) is deteriorating for greater frequencies. We can see that for the presented nanocavity sensors, Eq. (B5) is a good approximation.

get

$$
y^{\prime \prime}(s)-\frac{y^{\prime}(s)}{s+r_{0}}-\frac{a}{b} y(s)=0 .
$$

The substitution $x=i \sqrt{a / b}\left(s+r_{0}\right)$ leads to the slightly modified Bessel equation

$$
x^{2} \frac{d^{2} y}{d x^{2}}-x \frac{d y}{d x}+x^{2} y=0
$$

solved by $y(x)=x\left[c_{1} J_{1}(x)+c_{2} Y_{1}(x)\right]$, where $J_{n}$ and $Y_{n}$ are Bessel functions of the first and second kinds of order $n$. Putting everything together, with $d y / d x=x\left[c_{1} J_{0}(x)+\right.$ $\left.c_{2} Y_{0}(x)\right]$, we get

$$
z(s)=\frac{\sqrt{a b} \backslash i}{s+r_{0}} \frac{J_{0}(x(s))+\alpha Y_{0}(x(s))}{J_{1}(x(s))+\alpha Y_{1}(x(s))} \quad \text { with } \alpha=c_{2} / c_{1} .
$$

To fix the constant $\alpha$ a boundary condition is necessary. In the limit

$$
z(s \rightarrow 0)=\frac{\sqrt{a b}\left[I_{0}\left(\sqrt{\frac{a}{b}} r_{0}\right)+\alpha Y_{0}\left(i \sqrt{\frac{a}{b}} r_{0}\right)\right]}{r_{0}\left[I_{1}\left(\sqrt{\frac{a}{b}} r_{0}\right)-i \alpha Y_{1}\left(i \sqrt{\frac{a}{b}} r_{0}\right)\right]},
$$

where $I_{n}(x)=i^{-n} J_{n}(i x)$, the condition of a vanishing denominator fixes $\alpha$ so that we obtain the final result which is given in Eq. (6).

[1] E. Neher and B. Sakmann, Nature (London) 260, 799 (1976).

[2] S. Demarche, K. Sugihara, T. Zambelli, L. Tiefenauer, and J. Vörös, Analyst 136, 1077 (2011).

[3] W. L. C. Rutten, Annual Review of Biomedical Engineering 4, 407 (2002).

[4] I. L. Jones, P. Livi, M. Lewandowska, M. Fiscella, B. Roscic, and A. Hierlemann, Analytical and Bioanalytical Chemistry 399, 2313 (2011).

[5] J. Pine, J. Neurosci. Methods 2, 19 (1980).

[6] G. W. Gross, E. Rieske, G. W. Kreutzberg, and A. Meyer, Neuroscience Letters 6, 101 (1977).

[7] A. Stett, U. Egert, E. Guenther, F. Hofmann, T. Meyer, W. Nisch, and H. Haemmerle, Analytical and Bioanalytical Chemistry 377, 486 (2003).

\section{APPENDIX B: ASYMPTOTIC BEHAVIOR}

To study the asymptotic behavior of the solution Eq. (6), we rewrite it in units of $a$ and in the parameters $\lambda(\omega)=$ $\sqrt{\frac{a}{b(\omega)}}\left(r_{0}+s\right)$ and $v=\frac{r_{0}}{r_{0}+s}$, which are both dimensionless

$$
Z_{\mathrm{cav}}(s)=a \frac{-i I_{1}(\nu \lambda) Y_{0}(i \lambda)+I_{0}(\lambda) Y_{1}(i v \lambda)}{\lambda\left(-I_{1}(\nu \lambda) Y_{1}(i \lambda)+I_{1}(\lambda) Y_{1}(i v \lambda)\right.} .
$$

Given $\lambda \ll 1$, for small resistivities and not too large frequencies, we can linearize the equation. The limit $\lambda \rightarrow 0$ (for $\nu$ not necessarily small) is

$$
\begin{aligned}
Z_{\mathrm{cav}}(\lambda \ll 1) & =a\left(\frac{2}{\left(1-v^{2}\right) \lambda^{2}}+O(1)\right) \\
& \simeq \frac{2 b}{2 r_{0} s+s^{2}} \\
& =\frac{1}{\pi\left(2 s r_{0}+s^{2}\right) C_{h}(i \omega)^{\beta}} .
\end{aligned}
$$

The approximately equal sign indicates that we neglect the $O$ (1) term. The result is what we would expect as impedance from an electrode ring with area $A=\pi\left[\left(r_{0}+s\right)^{2}-r_{0}^{2}\right]=$ $\pi\left(2 r_{0} s+s^{2}\right)$. If the radius of the electrode $s+r_{0}$ is large compared to the aperture $r_{0}, v$ is small (for $\lambda$ not necessarily small). In this case

$$
Z_{\text {cav }}(v \ll 1)=a\left(\frac{I_{0}(\lambda)}{\lambda I_{1}(\lambda)}+O\left(v^{2}\right)\right) \simeq \frac{a I_{0}(\lambda)}{\lambda I_{1}(\lambda)} .
$$

Using the parameters from Fig. 2 and typical angular frequencies of $\omega \in[0,2 \pi \times 10000 \mathrm{~Hz}] \simeq\left[0,60000 \frac{1}{s}\right]$ for

$$
\lambda=\sqrt{\frac{a}{b(\omega)}}\left(r_{0}+s\right)=\sqrt{2 \pi a C_{h}(i \omega)^{\beta}}\left(r_{0}+s\right)
$$

we get that $|\lambda| \in[0,3]$. Also $v \approx 0.2$, which indicates that for small frequencies, both approximations are comparable. For greater frequencies, Eq. (B5) should be the better approximation. In Fig. 7 we compare Eqs. (B4) and (B5) with the full solution as a function of $f=\omega / 2 \pi$. The error measure shown is

$$
E=\frac{\left|Z_{\mathrm{cav}}\right|-\left|Z_{\mathrm{cav}}^{\mathrm{appr}}\right|}{\left|Z_{\mathrm{cav}}\right|},
$$

where $Z_{\text {cav }}$ is the full solution, and $Z_{\text {cav }}^{\text {appr }}$ are the two approximative solutions. This figure confirms our conclusion.
[8] J. K. Y. Law, C. K. Yeung, B. Hofmann, S. Ingebrandt, J. A. Rudd, A. Offenhäusser, and M. Chan, Physiol. Meas. 30, 155 (2009).

[9] G. Shahaf and S. Marom, The Journal of Neuroscience 21, 8782 (2001).

[10] R. Segev, Y. Shapira, M. Benveniste, and E. Ben-Jacob, Phys. Rev. E 64, 011920 (2001).

[11] A. Gramowski, K. Jügelt, D. G. Weiss, and G. W. Gross, European Journal of Neuroscience 19, 2815 (2004).

[12] G. W. Gross, A. Harsch, B. K. Rhoades, and W. Göpel, Biosens. Bioelectron. 12, 373 (1997).

[13] B. C. Wheeler and G. J. Brewer, Proc. IEEE 98, 398 (2010).

[14] A. Hierlemann, U. Frey, S. Hafizovic, and F. Heer, Proc. IEEE 99, 252 (2011). 
[15] L. Berdondini, K. Imfeld, A. Maccione, M. Tedesco, S. Neukom, M. Koudelka-Hep, and S. Martinoia, Lab Chip 9, 2644 (2009).

[16] Z. Yu, T. E. McKnight, M. N. Ericson, A. V. Melechko, M. L. Simpson, and B. Morrison, Nano Letters 7, 2188 (2007).

[17] T. Gabay, M. Ben-David, I. Kalifa, R. Sorkin, Z. R. Abrams, E. Ben-Jacob, and Y. Hanein, Nanotechnology 18, 035201 (2007).

[18] Y. Hanein, Phys. Status Solidi B 247, 2635 (2010).

[19] D. Brüggemann, B. Wolfrum, V. Maybeck, Y. Mourzina, M. Jansen, and A. Offenhäusser, Nanotechnology 22, 265104 (2011).

[20] J.-H. Kim, G. Kang, Y. Nam, and Y.-K. Choi, Nanotechnology 21, 085303 (2010).

[21] P. Verma and N. A. Melosh, Appl. Phys. Lett. 97, 033704 (2010).

[22] A. Hai, D. Kamber, G. Malkinson, H. Erez, N. Mazurski, J. Shappir, and M. E. Spira, Journal of Neural Engineering 6, 066009 (2009).

[23] E. Kätelhön, B. Hofmann, S. G. Lemay, M. A. G. Zevenbergen, A. Offenhäusser, and B. Wolfrum, Anal. Chem. 82, 8502 (2010).

[24] B. Hofmann, E. Kätelhön, M. Schottdorf, A. Offenhäusser, and B. Wolfrum, Lab Chip 11, 1054 (2011).

[25] A. Hassibi, R. Navid, R. W. Dutton, and T. H. Lee, J. Appl. Phys 96, 1074 (2004).

[26] N. Joye, A. Schmid, and Y. Leblebici, Neurocomputing 73, 250 (2009).

[27] P. Verma, I. Y. Wong, and N. A. Melosh, Biointerphases 5, 37 (2010).

[28] R. Weis, B. Müller, and P. Fromherz, Phys. Rev. Lett. 76, 327 (1996).

[29] P. Fromherz, Eur. Biophys. J. 28, 254 (1999).

[30] R. Weis and P. Fromherz, Phys. Rev. E 55, 877 (1997).

[31] M. Pabst, G. Wrobel, S. Ingebrandt, F. Sommerhage, and A. Offenhäusser, Eur. Phys. J. E 24, 1 (2007).

[32] W. C. Claycomb, N. A. Lanson, B. S. Stallworth, D. B. Egeland, J. B. Delcarpio, A. Bahinski, and N. J. Izzo, Proc. Natl. Acad. Sci. 95, 2979 (1998).
[33] C. Sprössler, M. Denyer, S. Britland, W. Knoll, and A. Offenhäusser, Phys. Rev. E 60, 2171 (1999).

[34] S. Ingebrandt, C.-K. Yeung, M. Krause, and A. Offenhäusser, Biosens. Bioelectron. 16, 565 (2001).

[35] W. G. Regehr, J. Pine, C. S. Cohan, M. D. Mischke, and D. W. Tank, J. Neurosci. Methods 30, 91 (1989).

[36] P. Fromherz, Ann. N.Y. Acad. Sci. 1093, 143 (2006).

[37] S. Haruo, J. Theor. Biol. 30, 59 (1971).

[38] R. W. Joyner, F. Ramon, and J. W. Morre, Circulation Research 36, 654 (1975).

[39] H. Shiba and Y. Kanno, Radiat. Environ. Biophys. 7, 295 (1971).

[40] R. W. de Boer and A. van Oosterom, Medical and Biological Engineering and Computing 16, 1 (1978).

[41] E. T. McAdams, A. Lackermeier, J. A. McLaughlin, D. Macken, and J. Jossinet, Biosens. Bioelectron. 10, 67 (1995).

[42] J. B. Bates, Y. T. Chu, and W. T. Stribling, Phys. Rev. Lett. 60, 627 (1988).

[43] S. H. Liu, Phys. Rev. Lett. 55, 529 (1985).

[44] R. Kant, R. Kumar, and V. K. Yadav, J. Phys. Chem. C 112, 4019 (2008).

[45] A. L. Hodgkin and A. F. Huxley, Am. J. Physiol. 117, 500 (1952).

[46] A. Nygren, C. Fiset, L. Firek, J. W. Clark, D. S. Lindblad, R. B. Clark, and W. R. Giles, Circulation Research 82, 63 (1998).

[47] S. Pelloux, J. Robillard, R. Ferrera, A. Bilbaut, C. Ojeda, V. Saks, M. Ovize, and Y. Tourneur, Progress in Biophysics and Molecular Biology 90, 270 (2006).

[48] A. Cohen, J. Shappir, S. Yitzchaik, and M. E. Spira, Biosens. Bioelectron. 23, 811 (2008).

[49] A. Hai, A. Dormann, J. Shappir, S. Yitzchaik, C. Bartic, G. Borghs, J. P. M. Langedijk, and M. E. Spira, Journal of the Royal Society Interface 6, 1153 (2009).

[50] E. L. Ince, Ordinary Differential Equations (Dover, New York, 1956). 